\title{
1 The role of neuropeptides in learning: insights from C. elegans
}

2 Nathan De Fruyt ${ }^{1}$, Alex J Yu ${ }^{2}$, Catharine H Rankin²,3, Isabel Beets ${ }^{1}$, Yee Lian Chew ${ }^{4, *}$

3 1- Department of Biology, KU Leuven, Leuven 3000 Belgium

4 2- Djavad Mowafaghian Centre for Brain Health, University of British Columbia, Vancouver,

5 BC V6T2B5 Canada

6 3- Department of Psychology, University of British Columbia, Vancouver, BC V6T1Z4

7 Canada

8 4- Molecular Horizons and School of Chemistry and Molecular Bioscience, University of

9 Wollongong and Illawarra Health and Medical Research Institute, NSW 2522 Australia

$10 *$ - to whom correspondence should be addressed: ylchew@,uow.edu.au

12 Abstract (150 words)

13 Learning is critical for survival as it provides the capacity to adapt to a changing environment.

14 At the molecular and cellular level, learning leads to alterations within neural circuits that

15 include synaptic rewiring and synaptic plasticity. These changes are mediated by signalling

16 molecules known as neuromodulators. One such class of neuromodulators are neuropeptides,

17 a diverse group of short peptides that primarily act through $\mathrm{G}$ protein-coupled receptors. There

18 has been substantial progress in recent years on dissecting the role of neuropeptides in learning

19 circuits using compact yet powerful invertebrate model systems. We will focus on insights

20 gained using the nematode Caenorhabditis elegans, with its unparalleled genetic tractability,

21 compact nervous system of $\sim 300$ neurons, high level of conservation with mammalian systems and amenability to a suite of behavioural analyses. Specifically, we will summarise recent 
1 discoveries in C. elegans on the role of neuropeptides in non-associative and associative

2 learning.

3 Keywords-neuropeptides; C. elegans; associative learning; non-associative learning

\section{$5 \quad$ Introduction}

6 Learning is a vital property that allows animals to adapt behaviour to a continuously changing 7 environment. At a cellular level, learning leads to changes within neural circuits. These include 8 rewiring of synaptic connections, alteration of the amount of presynaptically released 9 neurotransmitters as well as changes in the number and sensitivity of postsynaptic receptors.

10 Such processes are modulated by non-autonomous signals called neuromodulators ${ }^{1}$. In contrast 11 to classical neurotransmitters released from synaptic vesicles, many neuromodulators are 12 "extrasynaptically" released and transported across larger distances by volume transmission, 13 generating auto-, para- or even endocrine effects.

15 One of the largest and most diverse groups of neuromodulators is represented by neuropeptides, 16 short peptides ( $\sim 3-100$ amino acids) that primarily bind to the similarly diverse group of $\mathrm{G}$ 17 protein-coupled receptors (GPCRs). Because of this diversity, neuropeptide-receptor signalling 18 offers a rich versatility for the modulation of neural circuits ${ }^{2,3}$. Neuropeptide signals act on a 19 range of spatiotemporal scales to shape neural circuit activity, from single neurons to a multitude of tissues, within tightly controlled timeframes to functioning throughout an

21 organism's lifetime ${ }^{4,5}$. Indeed, neuropeptides play invaluable roles in shaping plastic 22 behaviours, including learning and memory, across diverse species. 
1 Despite substantial progress in illuminating neuropeptide functions in learning circuits, we are

2 only beginning to understand the underlying mechanisms. Improved tools to study localisation,

3 biochemical identity and function of peptides/receptors provide new opportunities to dissect

4 peptidergic modes of action in learning circuits within a living animal. Indeed, invertebrate

5 learning circuits have proven to be powerful models due to their compact size, genetic

6 tractability and amenability to a sophisticated technological toolbox including automated

7 behavioural analyses. In this regard, the nematode Caenorhabditis elegans stands out with a

8 highly compact nervous system of $\sim 300$ neurons, for which the anatomical connectome (the

9 network of synaptic connections) has been extensively described ${ }^{6}$. Here, we will summarise

10 main insights into neuropeptidergic signalling in C. elegans learning. We will first briefly

11 discuss new advances in understanding the conservation of neuropeptide systems, before

12 elaborating on the role of neuropeptides in two categories of implicit memory: non-associative

13 and associative learning

15 Conservation of Neuropeptide Signalling Systems

16 Neuropeptides are a diverse class of modulators present in all known metazoans ${ }^{3,7}$. However,

17 their short amino acid sequence has posed a challenge in assessing neuropeptide phylogeny and evolutionary history. In addition, GPCRs display high degrees of mosaicism due to many duplication events that complicate our understanding of their classification ${ }^{8}$. Recently, longrange phylogenetic analyses have gained traction due to the increased availability of published genomic sequences, which improve the quality of large-scale analyses. This has been accelerated by the development of improved algorithms to mine these resources. 
1 Besides neuropeptides, conserved neuropeptide receptors and neuropeptide processing

2 machinery are found in all bilaterians, implicating an ancient evolutionary origin for

3 neuropeptidergic signalling ${ }^{3,7,9}$. This has been corroborated by several independent large-scale

4 phylogenetic studies. These studies showed that $\sim 30$ peptidergic signalling systems were

5 present in the last common urbilaterian ancestor, before the divergence between protostome

6 and deuterostome lineages ${ }^{3,7}$.

8 The C. elegans genome encodes an estimated 150 neuropeptide GPCRs. It also contains at least

9153 neuropeptide-encoding genes that give rise to over 300 predicted bioactive peptides, many

10 of which have been biochemically confirmed ${ }^{9-11}$. The C. elegans genome encodes orthologs

11 of many evolutionarily ancient neuropeptidergic systems ${ }^{3,7}$. These include orthologs of the

12 oxytocin/vasopressin, gonadotropin-releasing hormone (GnRH), thyrotropin-releasing

13 hormone (TRH), neuromedin U (NMU), tachykinin, myoinhibitory peptide (MIP), and

14 neuropeptide Y (NPY) families $3,5,7,10,12,13$. For several neuropeptide systems, ligand-receptor

15 interactions between orthologous neuropeptides and predicted GPCRs have been confirmed

16 biochemically through receptor activation assays in cultured cells. For several of these,

17 structural homology appears to correlate with functional similarity.

19 Neuropeptides in non-associative learning in C. elegans

20 Non-associative learning is the simplest form of learning and has been ubiquitously observed

21 in animal species across evolution. Habituation and sensitization are two types of non-

22 associative learning: habituation is the response decrement as a result of being repeatedly

23 stimulated, whereas sensitization is the response facilitation produced by a novel and/or noxious stimulus. Habituation is conventionally framed as learning to ignore recurring stimuli 
1 that do not provide biologically relevant information, while sensitization is framed as

2 enhancing the robustness of responses to appetitive and aversive stimuli. Non-associative

3 learning in C. elegans was first demonstrated by Rankin et al. in $1990^{14}$. Since then, the field

4 has generated a rich body of literature on the cellular and molecular mechanisms of learning in

5 C. elegans (reviewed in ${ }^{15}$ ). Here, we will describe highlights from several recent studies

6 implicating neuropeptide signalling pathways in aspects of non-associative learning.

8 The FMRFamide-related neuropeptides encoded by the $f l p-20$ gene have been shown to play

9 key roles in both habituation and sensitization. Li et al. (2013) found that FLP-20 neuropeptides

10 released from the mechanosensory touch receptor neurons (TRNs) were required for a massed

11 training-induced, intermediate-term memory (lasting $12 \mathrm{hrs}$ ) for mechanosensory habituation.

12 Interestingly, there is no evidence that FLP-20 is involved in either short-term or long-term habituation, as flp-20 mutants formed normal short-term and long-term memories for habituation. These findings indicate that neuropeptides mediate specific phases of nonassociative memory ${ }^{16}$. FLP-20 is also critical for sensitization. Chew et al. (2018) investigated the cellular and molecular pathways underlying mechanosensory-induced sensitization of an optogenetically elicited nociceptive response in the polymodal neuron $\mathrm{ASH}^{17}$. In this paradigm, mechanosensory stimulation enhanced the calcium transient in response to natural stimuli $(0.5$ $\mathrm{M}$ glycerol) in $\mathrm{ASH}$, as well as the magnitude of the escape response (backwards movement/reversal) to optogenetic stimulation of $\mathrm{ASH}$, and touch receptor neuron-released FLP-20 mediated this effect. Intriguingly, in these two reports FLP-20 mediates two opposite

22 behavioural changes, response decrement and response facilitation. One possible explanation for this is that FLP-20 neuropeptides act on different cellular and/or molecular signalling components to mediate different types of non-associative learning. In intermediate-term mechanosensory habituation, the memory is correlated with a flp-20-dependent increase in the 
1 presynaptic vesical density in the PLM mechanosensory neurons, presumably increasing

2 neurotransmitter release by recruiting more synaptic vesicles to the presynaptic terminals ${ }^{16}$.

3 On the other hand, mechanosensory-induced ASH sensitization requires FLP-20 signalling

4 through the neuropeptide receptor FRPR-3, and one of its major sites of action, the

5 neuroendocrine neuron RID, was identified ${ }^{17}$. Taken together, these studies show that the same

6 neuropeptides released by the touch receptor neurons can produce both habituation and

7 sensitization (Figure 1).

A

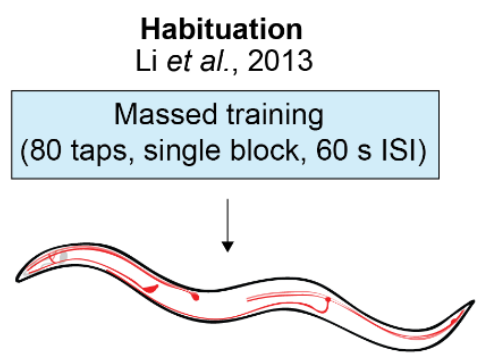

Release of FLP-20 from touch receptor neurons

Intermediate term (12 h) memory for habituation
B Sensitisation

Chew et al., 2018

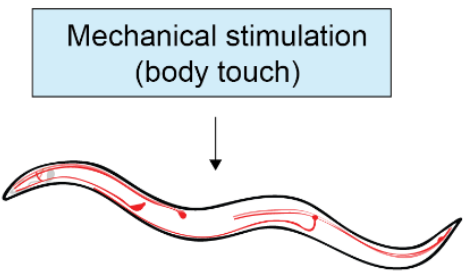

Release of FLP-20 from touch receptor neurons

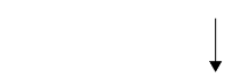

Activation through RID (+others) through FRPR-3

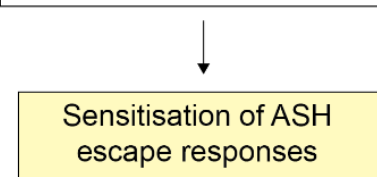

C

Touch receptor neurons

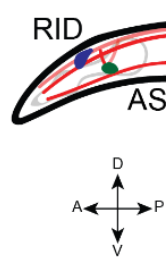

Touch receptor neurons

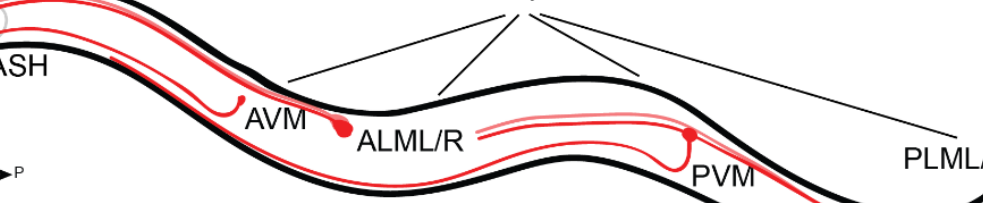

9

10 Figure 1: The neuropeptide FLP-20 is required for both habituation and sensitization. A)

11 Intermediate-term memory for habituation in response to mechanical stimulation (taps) after

12 massed training requires FLP-20 release from the touch receptor neurons (TRNs). ISI:

13 Interstimulus interval. B) Sensitization of escape responses mediated by the nociceptive neuron 
1 ASH requires FLP-20 release from the touch neurons, which act through the FRPR-3 receptor

2 to activate the neuroendocrine cell RID. C) Schematic of specific neurons highlighted above: six TRNs (ALML/R, AVM, PVM, PLML/R) in the body and tail, the RID interneuron and

4 ASH sensory neuron. For simplicity, only the soma of RID and ASH are shown.

6 The role of another class of neuropeptides, Pigment-Dispersing Factor (PDF) peptides, which 7 are functional homologs of the vertebrate vasoactive intestinal peptide (VIP) $)^{18,19}$, was revealed 8 in studies of ASH-mediated nociceptive response habituation. In C. elegans, the $p d f-1$ and $p d f-$ 92 genes encode the two PDF ligands, and the $p d f r$ - 1 gene encodes the receptor. Repeated ASH optogenetic stimulation produces two sets of coordinated behavioural changes: habituation of 11 the ASH-mediated escape response, and sensitization of forward locomotion ${ }^{20}$. These simultaneous changes may act together to trigger animals' dispersal away from where the nociceptive stimulus was received. The coordination of the two forms of plasticity is mediated by PDF signalling, as $p d f-1$ and $p d f-2$ mutants, as well as the $p d f r-1$ receptor mutant, were defective in these coordinated behaviours. Interestingly, the sites of action of PDF signalling appear to be distributed across the nervous system and body wall muscle, as selectively reexpressing $p d f r-1$ in either neurons or the muscle tissues in a $p d f r-1$ mutant partially restored the behavioural effects. Thus, in this case, the PDF neuropeptides regulate the coordination between response habituation and ongoing locomotion sensitization, to adaptively shift the behavioural strategy to promote dispersal. These effects are summarised in Table 1.

22 Neuropeptides have also been shown to interact with other neuromodulators to mediate nonassociative learning. C. elegans naturally avoid repulsive odours, for example, 2-nonanone, and the avoidance response to this volatile repellent can be sensitised if worms are pre-exposed 
1 to the same odour ${ }^{21}$. Animals with mutations in genes encoding key components of the

2 neuropeptide biosynthesis machinery, egl-3 (proprotein convertase) and egl-21

3 (carboxypeptidase N), failed to show this increased avoidance. Although neuropeptide

4 synthesis is generally required for this learned avoidance response, it is unclear which specific

5 neuropeptides are responsible. Interestingly, although dopamine is not involved in the

6 sensitization, it is critical for maintaining the direction of avoidance away from the source of

7 noxious stimuli. Taken together, neuropeptides and dopamine work together to increase the

8 effectiveness of the learned avoidance response.

10 To summarise, the role of neuropeptides in non-associative learning is highlighted in three key

11 findings: (1) Neuropeptides mediate different types of non-associative learning through 12 different pathways, likely governed by the specific types of neuropeptide receptors expressed 13 in different cells and tissues. (2) Neuropeptides are important for multi-component, coordinated

14 behavioural changes in non-associative learning. (3) Neuropeptides interact with other 15 neuromodulators to shape the response patterns in learning.

\section{$17 \quad$ Neuropeptides in associative learning in $\mathrm{C}$. elegans}

18 Associative learning refers to processes where an association is formed between two stimuli. C. elegans has been used to study short-, intermediate- and long-term memory formation, as well as both positive and negative associative learning using a multitude of conditioned stimuli,

21 including gustatory, olfactory, thermal, mechanical and gaseous cues, or more complex stimuli 22 encoding a combination of multiple cues, such as the presence of pathogens or food. Several 23 conserved neuropeptidergic systems have been shown to play a role in C. elegans associative 24 learning, including homologs for insulin (INS-1) and insulin-like peptides (ILPs), 
1 oxytocin/vasopressin (NTC-1), myoinhibitory peptide (MIP-1/NLP-38), neuromedin U

2 (CAPA-1/NLP-44), elevenin (SNET-1), short neuropeptide F receptor signalling (NPR-1), and

3 PDF-1 signaling (also mentioned above for non-associative learning) 4,5,10,12,22-28. These effects

4 are summarised in Table 1.

5

6 The specific effects of peptidergic signalling on associative learning often vary between

7 different neuropeptides. While some $C$. elegans neuropeptides have been found to impact a

8 wide diversity of learning behaviours, others have shown to modulate only specific associative

9 learning circuits. For example, MIP signalling is required for gustatory aversive learning - to

10 form a negative association between salt and the absence of food in the environment - but not

11 for learning positive associations between the same salt cue and the presence of food ${ }^{10}$. In contrast, insulin-like signalling via the insulin receptor homolog DAF-2 appears to have an

13 effect on both positive and negative associative learning 4,22,26,29. Remarkably, insulin

14 signalling has opposing effects on positive and negative associations: daf-2 mutants fail to form

15 a negative association when a conditioned stimulus is paired with starvation, whereas in

16 positive associative learning paradigms (conditioned stimulus paired with appetitive cue), daf-

172 mutants show enhanced learning.

19 Neuropeptidergic signalling is not necessarily restricted to a specific conditioning cue either, 20 as one neuropeptide can have modulatory effects on a variety of associative learning behaviours

21 (Figure 2A) ${ }^{4,23,30,31}$. On the other hand, a specific learned behaviour can be modulated by a 22 multitude of neuropeptides. For example, gustatory associative learning in C. elegans is 23 modulated by at least four neuropeptides, including MIP-1, CAPA-1, NTC-1 and INS-1

24 (Figure 2B $)^{5,10,12,31}$. At least five neuropeptides have also been shown to modulate learned 
1 pathogen avoidance in response to environmental cues (INS-4, INS-16, INS-6, INS-7 and INS-

$211)^{22,24-26}$.

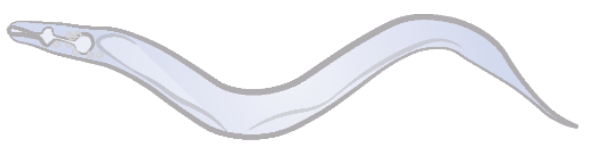

Associative learning in C. elegans
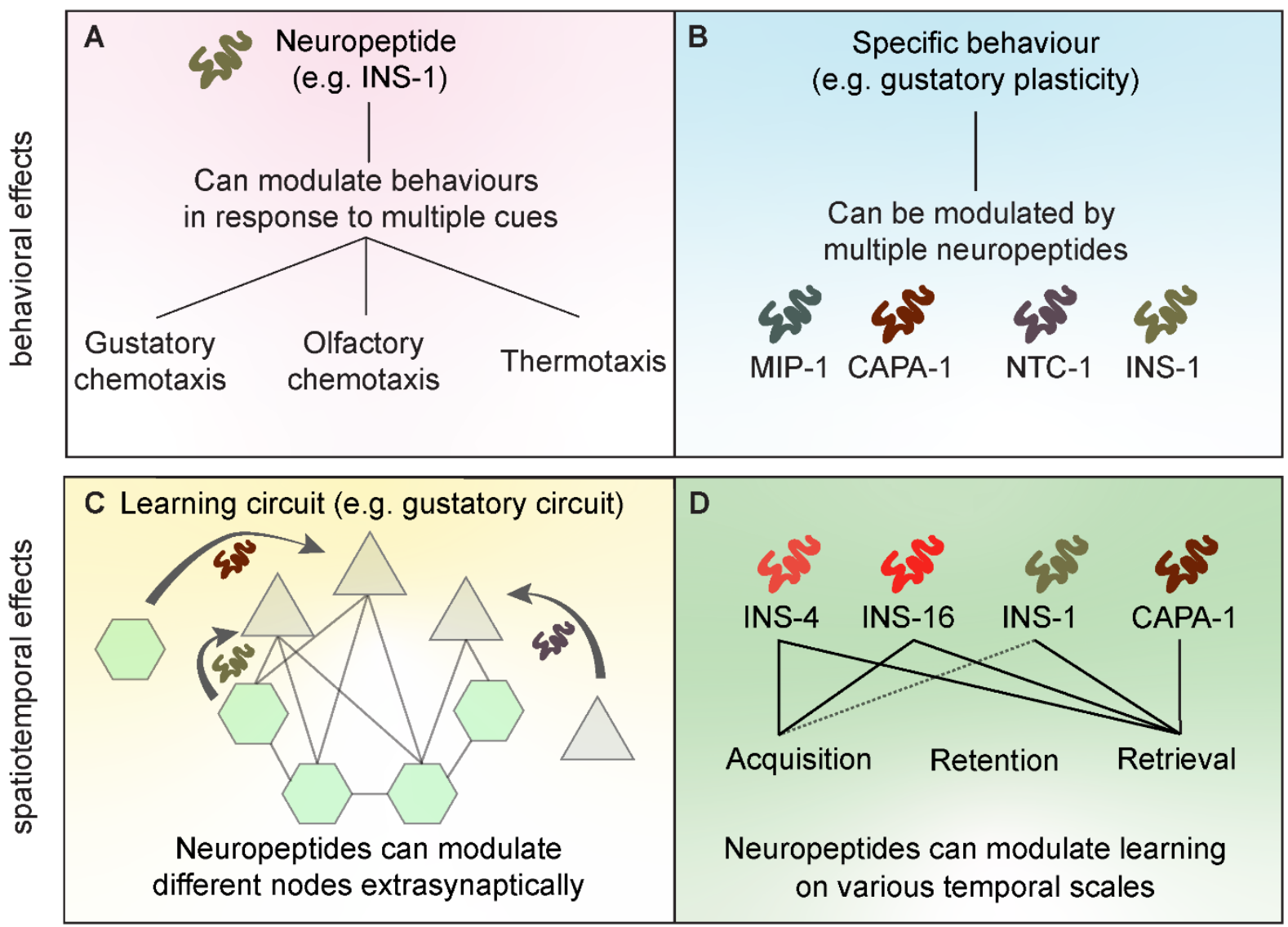

4 Figure 2: Studies of neuropeptide signalling in associative learning in C. elegans indicate that

5 A) one neuropeptide can have modulatory effects on a variety of learned behaviours; B) a

6 specific learned behaviour can be modulated by multiple neuropeptides; C) learning circuits

7 are often modulated at different nodes by different neuropeptides; D) neuropeptides can

8 differentially modulate specific stages of the learning process.

9 In such complex networks of neuropeptidergic modulation, it remains a challenge to unravel

10 the individual effects of each of these modulatory systems on the learning circuit. Recent work reveals that the roles of individual neuropeptides in associative learning circuits can in part be 
1 deduced by restricted spatiotemporal effects of neuropeptidergic signalling. Most

2 neuropeptides have been found to act extrasynaptically ${ }^{2}$. This "wireless" mode of signalling

3 can bypass restrictions in neural communication imposed by the anatomical connectome.

4 Neuropeptides can thereby modulate diverse neurons in the learning circuit that do not have to

5 be synaptically connected to the peptidergic cell (Figure 2C). Most examples of

6 neuropeptidergic modulation in associative learning circuits so far illustrate modulation at the

7 level of sensory neurons and interneurons. For example, INS-6 and INS-7 modulate pathogen

8 avoidance learning by signalling between two pairs of sensory neurons that share no synaptic

9 connections (ASI and URX) and a pair of interneurons (RIA) ${ }^{6,22}$. Likewise, NMU-like CAPA-

101 neuropeptides signal extrasynaptically from sensory ASG neurons to another pair of sensory neurons (AFD) to modulate gustatory associative learning. When multiple neuropeptides modulate a specific learning circuit, such as in the case of gustatory aversive learning, different neuropeptide signals have been found to act on distinct nodes within the circuit (Figure 2C).

14 For example, the neuropeptides INS-1, NTC-1 and CAPA-1 are all required for gustatory aversive learning, yet modulate distinct targets in the gustatory circuit, i.e. ASER, ASEL and AFD neurons $5,12,31$. Such concerted action of neuropeptides on different nodes in the learning circuit could be important to further tune learning behaviour to the animal's physiological needs ${ }^{26}$. INS-1 modulates gustatory aversive learning independently from other neuropeptides (e.g. MIP-1), indicating that different neuropeptides likely act in parallel to modulate 20 learning ${ }^{10}$.

22 Synaptic plasticity and altered neuronal activity are key cellular events during learning that are subject to neuropeptidergic regulation; however, the precise modulatory mechanisms are not completely understood. Indeed, it appears that the effects of neuropeptides on synaptic plasticity are not generalisable. For example, associative gustatory aversive learning, which 
1 requires INS-1 signalling, increases calcium activity and decreases synaptic release from the

2 ASER salt-sensory neuron ${ }^{29}$. In contrast, learning-induced changes in calcium activity of the

3 ASE neurons are not affected by CAPA-1 neuropeptide signalling, even though capa-1 is

4 required for learning ${ }^{5}$.

6 Besides targeted spatial effects, it has become clear that different neuropeptidergic pathways

7 can modulate distinct temporal phases of C. elegans associative learning. Learning comprises

8 at least three differentially modulated stages: memory acquisition, retention and retrieval; each

9 of which can be separately modulated (Figure 2D). During olfactory associative learning in $C$.

10 elegans, INS-1 signalling through DAF-2 is required at the retrieval stage, but INS-1 only

11 partially contributes to acquisition of these memories ${ }^{4,23}$. Optogenetic manipulation of CAPA-

12 1-expressing neurons in the gustatory associative learning circuit showed that CAPA-1 signalling is only required for the retrieval, but not the acquisition, of learned salt avoidance ${ }^{5}$.

14 Importantly, the time window in which neuropeptides act differs between individual signals.

15 In contrast to the stage-specific effects of CAPA-1 and INS-1, INS-4 and INS-16 are required 16 in both acquisition and retrieval stages of learned pathogen avoidance behaviour ${ }^{26}$.

18 Memory is traditionally also categorised as short-, intermediate- or long-term, each of which has specific molecular and behavioural hallmarks. Neuropeptides differentially affect aspects of short- and long-term associative memory formation in C. elegans ${ }^{10,23}$. For example, while 21 MIP signalling was shown to similarly modulate short- and long-term gustatory associative 22 memory, DAF-2 insulin-like receptor signalling differentially affects short- and long-term 23 olfactory associative memory ${ }^{10,23}$. Investigations into the effects of single neuropeptides on 24 both short- and long-term associative memory in C. elegans are limited to these examples. 
1 However, several neuropeptide genes are upregulated after both appetitive and aversive long-

2 term associative learning, suggesting a role in long-term memory processes ${ }^{32,33}$. In line with

3 these findings, evidence from other vertebrate and invertebrate models suggests that

4 differential neuropeptidergic modulation of short- and long-term memory may be a general

5 feature in associative memory formation. 
1 Table 1: Examples of neuropeptides and neuropeptide receptors modulating non-associative and associative learning in C. elegans. A question 2 mark indicates neuropeptide systems for which the cellular focus remains unknown. Under "Effect", upward arrows ( $\uparrow$ ) denote neuropeptide 3 systems that promote learning, where two upward arrows $(\uparrow \uparrow)$ indicate neuropeptide systems for which loss-of-function results in abolished 4 learning and a single upward arrow $(\uparrow)$ denotes a reduced ability to learn upon impaired peptidergic signalling. Downward arrows $(\downarrow)$ indicate 5 suppressive roles for neuropeptidergic signalling in learning. $\mathrm{TRN}=$ touch receptor neuron. $\mathrm{STM}=$ short-term memory, $\mathrm{LTM}=$ long-term memory.

\begin{tabular}{|c|c|c|c|c|c|c|}
\hline $\begin{array}{l}\text { Neuropeptide } \\
\text { gene }\end{array}$ & $\begin{array}{l}\text { Neuropeptide } \\
\text { receptor gene }\end{array}$ & Neurons (from $\rightarrow$ to) & Effect & Paradigm & Stage & Ref(s) \\
\hline \multicolumn{7}{|c|}{ Non-associative learning } \\
\hline \multirow[t]{2}{*}{ flp-20 } & frpr-3 & TRNs $\rightarrow$ RID & $\uparrow$ & mechanosensory-induced ASH sensitization & - & 17 \\
\hline & $?$ & TRNs & $\uparrow \uparrow$ & intermediate-term tap habituation (massed training with 80 taps) & - & 16 \\
\hline $\begin{array}{l}p d f-1 \\
p d f-2\end{array}$ & $p d f r-1$ & $?$ & $\uparrow$ & $\begin{array}{l}\text { Integration of ASH response habituation and locomotion } \\
\text { sensitization }\end{array}$ & - & 18 \\
\hline \multicolumn{7}{|c|}{ Associative learning } \\
\hline$n t c-1$ & $n t r-1$ & $\mathrm{AVK} \rightarrow \mathrm{ASEL}$ & $\uparrow$ & gustatory plasticity $(\mathrm{NaCl}+$ food absence $)$ & - & 12 \\
\hline \multirow[t]{3}{*}{ mip-1 } & sprr-2 & $?$ & $\uparrow$ & gustatory plasticity (STM; $\mathrm{NaCl}+$ food absence) & - & 10 \\
\hline & & $?$ & $\uparrow$ & salt avoidance learning (LTM; $\mathrm{NaCl}+$ starvation) & - & 10 \\
\hline & & $?$ & none & salt concentration memory $(\mathrm{NaCl}+$ food $)$ & - & 10 \\
\hline capa-1 & nmur-1 & $\mathrm{ASG} \rightarrow \mathrm{AFD}$ & $\uparrow$ & gustatory plasticity $(\mathrm{NaCl}+$ food absence; $\mathrm{NaCl}+$ benzaldehyde) & retrieval $(\uparrow)$ & 5 \\
\hline \multirow[t]{7}{*}{ ins-1 } & $d a f-2 c$ & $\mathrm{AIA} \rightarrow \mathrm{ASER}$ & $\uparrow \uparrow$ & gustatory plasticity ( $\mathrm{NaCl}+$ food absence) & - & $10,29,31$ \\
\hline & $d a f-2$ & $?$ & $\downarrow$ & massed positive butanone association (STM; butanone + food) & - & 23 \\
\hline & & $?$ & $\downarrow$ & spaced positive butanone association (LTM; butanone + food $)$ & - & 23 \\
\hline & $d a f-2$ & ASI \& AIA $\rightarrow$ AWC & $\uparrow \uparrow$ & benzaldehyde-starvation learning (benzaldehyde + starvation) & $\begin{array}{l}\text { acquisition }(\uparrow) \\
\text { retrieval }(\uparrow \uparrow)\end{array}$ & 4 \\
\hline & & $\mathrm{ASI} \& \mathrm{AIA} \rightarrow \mathrm{AWC}$ & $\uparrow \uparrow$ & butanone-starvation learning (butanone + starvation) & - & 4 \\
\hline & & ASI \& AIA $\rightarrow$ AWC & none & butanone enhancement (butanone + food) & - & 4 \\
\hline & $d a f-2$ & $?$ & $\downarrow$ & positive temperature learning (temperature + food) & - & 30 \\
\hline
\end{tabular}




\begin{tabular}{|c|c|c|c|c|c|c|}
\hline snet-1 & - & ASI \& ASK $\rightarrow ?$ & $\downarrow$ & benzaldehyde-starvation learning (benzaldehyde + starvation) & - & 27 \\
\hline$p d f-1$ & $p d f r-1$ & $\mathrm{MCM} \rightarrow ?$ & $\downarrow$ & gustatory plasticity $(\mathrm{NaCl}+$ food absence) & - & 28 \\
\hline ins-4 & $d a f-2$ & $\mathrm{AWA} \rightarrow ?$ & $\uparrow \uparrow$ & pathogen avoidance learning & $\begin{array}{l}\text { acquisition }(\uparrow \uparrow) \\
\text { retrieval }(\uparrow \uparrow)\end{array}$ & 26 \\
\hline ins-16 & $d a f-2$ & $\mathrm{ADL} \rightarrow ?$ & $\downarrow \downarrow$ & pathogen avoidance learning & $\begin{array}{l}\text { acquisition }(\downarrow \downarrow) ; \\
\text { retrieval }(\downarrow \downarrow)\end{array}$ & 26 \\
\hline ins-6 & $d a f-2$ & ASI $\rightarrow$ URX & $\uparrow \uparrow$ & pathogen avoidance learning & - & 22 \\
\hline ins-7 & daf-2 & $\mathrm{URX} \rightarrow \mathrm{RIA}$ & $\downarrow \downarrow$ & pathogen avoidance learning & - & 22 \\
\hline ins-11 & $d a f-2$ & Intestine $\rightarrow$ ADF,ASI & $\downarrow$ & pathogen avoidance learning & - & 24 \\
\hline
\end{tabular}




\section{Concluding remarks}

2 C. elegans is a highly effective model system in which to advance our understanding of the

3 cellular and circuit basis of learning. Technical advances in genetics, optogenetics, and

4 connectomics, as well as the potential for large-scale screens, provide a uniquely powerful

5 approach through which we can investigate the molecular foundation of learning behaviour.

6 Studying C. elegans learning behaviours thereby advanced our understanding of the pivotal

7 and various roles by which neuropeptides affect nearly every aspect of non-associative and

8 associative learning, on different spatiotemporal scales and interacting with a variety of

9 pathways and signals. Together with its amenability to high-throughput in vivo behavioural and

10 neurophysiological screening and new biochemical information on the interactions between

11 neuropeptides and GPCRs ${ }^{34}$, we expect that C. elegans will become an important model for

12 further research in the context of learning and memory disorders.

\section{Acknowledgements}

15 N.D.F and I.B. are funded by the Research Foundation - Flanders (grant G093419N) and KU

16 Leuven Research Fund (C14/15/049), A.J.Y. is funded by a Natural Sciences and Engineering

17 Research Council (NSERC) PGS-D Scholarship, C.H.R. is funded by the NSERC (RGPIN18 2019-05558) and Canadian Institutes of Health (CIHR PJT 165947). Y.L.C is funded by the

19 National Health and Medical Research Council (NHMRC) (APP1173448) and Rebecca L

20 Cooper Medical Research Foundation (PG2020652). 


\section{References:}

1 Dismukes, R. K. New concepts of molecular communications among neurons. The Behavioral and Brain Sciences 2, 409-448 (1979).

2 Bargmann, C. I. Beyond the connectome: How neuromodulators shape neural circuits. Bioessays 34, 458-465, doi:10.1002/bies.201100185 (2012).

3 Mirabeau, O. \& Joly, J. S. Molecular evolution of peptidergic signaling systems in bilaterians. Proceedings of the National Academy of Sciences 110, E2028-E2037, doi:10.1073/pnas.1219956110 (2013).

4 Lin, C. H. A. et al. Insulin signaling plays a dual role in Caenorhabditis elegans memory acquisition and memory retrieval. Journal of Neuroscience 30, 8001-8011, doi:10.1523/JNEUROSCI.4636-09.2010 (2010).

$5 \quad$ Watteyne, J. et al. Neuromedin $U$ signaling regulates retrieval of learned salt avoidance in a C. elegans gustatory circuit. Nature Communications 11, doi:10.1038/s41467-020-15964-9 (2020).

6 Cook, S. J. et al. Whole-animal connectomes of both Caenorhabditis elegans sexes. Nature 571, 63-71, doi:10.1038/s41586-019-1352-7 (2019).

7 Jekely, G. Global view of the evolution and diversity of metazoan neuropeptide signaling. Proceedings of the National Academy of Sciences 110, 8702-8707, doi:10.1073/pnas.1221833110 (2013).

8 Allaby, R. G. \& Woodwark, M. Phylogenomic analysis reveals extensive phylogenetic mosaicism in the human GPCR superfamily. Evolutionary Bioinformatics 3, 357-370, doi:10.1177/117693430700300002 (2007).

9 Van Bael, S. et al. Mass spectrometric evidence for neuropeptide-amidating enzymes in Caenorhabditis elegans. Journal of Biological Chemistry 293, doi:10.1074/jbc.RA117.000731 (2018).

10 Peymen, K. et al. Myoinhibitory peptide signaling modulates aversive gustatory learning in Caenorhabditis elegans. PLOS Genetics 15, e1007945-e1007945, doi:10.1371/journal.pgen.1007945 (2019).

11 Van Bael, S. et al. A Caenorhabditis elegans Mass Spectrometric Resource for Neuropeptidomics. Journal of the American Society for Mass Spectrometry 29, 879889, doi:10.1007/s13361-017-1856-z (2017).

12 Beets, I. et al. Vasopressin/Oxytocin-Related Signaling Regulates Gustatory Associative Learning in C. elegans. Science 338, 543-546 (2012).

13 Van Sinay, E. et al. Evolutionarily conserved TRH neuropeptide pathway regulates growth in Caenorhabditis elegans. PNAS, 4065-4074, doi:10.1073/pnas.1617392114 (2017).

14 Rankin, C. H., Beck, C. D. O. \& Chiba, C. M. Caenorhabditis elegans: a new model system for the study of learning and memory. Behav Brain Res 37, 89-92 (1990).

15 Ardiel, E. L. \& Rankin, C. H. An elegant mind: learning and memory in Caenorhabditis elegans. Learn Mem 17, 191-201, doi:10.1101/Im.960510 (2010).

$16 \mathrm{Li}, \mathrm{C}$. et al. The FMRFamide-related neuropeptide FLP-20 is required in the mechanosensory neurons during memory for massed training in C. elegans. Learn Mem 20, 103-108, doi:10.1101/Im.028993.112 (2013).

17 Chew, Y. L. et al. An Afferent Neuropeptide System Transmits Mechanosensory Signals Triggering Sensitization and Arousal in C. elegans. Neuron 99, 1233-1246 e1236, doi:10.1016/j.neuron.2018.08.003 (2018). 
18 Janssen, T. et al. Functional characterization of three $\mathrm{G}$ protein-coupled receptors for pigment dispersing factors in Caenorhabditis elegans. J Biol Chem 283, 15241-15249, doi:10.1074/jbc.M709060200 (2008).

19 Talsma, A. D. et al. Remote control of renal physiology by the intestinal neuropeptide pigment-dispersing factor in Drosophila. Proc Natl Acad Sci U S A 109, 12177-12182, doi:10.1073/pnas.1200247109 (2012).

20 Ardiel, E. L., Yu, A. J., Giles, A. C. \& Rankin, C. H. Habituation as an adaptive shift in response strategy mediated by neuropeptides. npj Science of Learning 2, 9, doi:10.1038/s41539-017-0011-8 (2017).

21 Yamazoe-Umemoto, A., Fujita, K., lino, Y., Iwasaki, Y. \& Kimura, K. D. Modulation of different behavioral components by neuropeptide and dopamine signalings in nonassociative odor learning of Caenorhabditis elegans. Neurosci Res 99, 22-33, doi:10.1016/j.neures.2015.05.009 (2015).

22 Chen, Z. et al. Two Insulin-like Peptides Antagonistically Regulate Aversive Olfactory Learning in C. elegans. Neuron 77, 572-585, doi:10.1016/j.neuron.2012.11.025 (2013).

23 Kauffman, A. L., Ashraf, J. M., Corces-Zimmerman, M. R., Landis, J. N. \& Murphy, C. T. Insulin Signaling and Dietary Restriction Differentially Influence the Decline of Learning and Memory with Age. PLoS biology 8, doi:10.1371/journal.pbio.1000372 (2010).

24 Lee, K. \& Mylonakis, E. An Intestine-Derived Neuropeptide Controls Avoidance Behavior in Caenorhabditis elegans. Cell Reports 20, 2501-2512, doi:10.1016/j.celrep.2017.08.053 (2017).

25 Singh, J. \& Aballay, A. Intestinal infection regulates behavior and learning via neuroendocrine signaling. eLIFE 8, 1-22 (2019).

26 Wu, T. et al. Pheromones Modulate Learning by Regulating the Balanced Signals of Two Insulin-like Peptides. Neuron 104, 1095-1109.e1095, doi:10.1016/j.neuron.2019.09.006 (2019).

27 Yamada, K. et al. Olfactory Plasticity Is Regulated by Pheromonal Signalin in Caenorhabditis elegans. Science 329, 1647-1650 (2010).

28 Sammut, M. et al. Glia-derived neurons are required for sex-specific learning in C. elegans. Nature 526, 385-390, doi:10.1038/nature15700 (2015).

29 Oda, S., Tomioka, M. \& lino, Y. Neuronal plasticity regulated by the insulin-like signaling pathway underlies salt chemotaxis learning in Caenorhabditis elegans. Journal of Neurophysiology 106, 301-308, doi:10.1152/jn.01029.2010 (2011).

30 Murakami, H., Bessinger, K., Hellmann, J. \& Murakami, S. Aging-Dependent and Independent Modulation of Associative Learning Behavior by Insulin / Insulin-Like Growth Factor-1 Signal in Caenorhabditis elegans. The Journal of Neuroscience 25, 10894-10904, doi:10.1523/JNEUROSCI.3600-04.2005 (2005).

31 Tomioka, M. et al. The insulin/PI 3-kinase pathway regulates salt chemotaxis learning in Caenorhabditis elegans. Neuron 51, 613-625, doi:10.1016/j.neuron.2006.07.024 (2006).

32 Freytag, V. et al. Genome-Wide Temporal Expression Profiling in Caenorhabditis elegans Identifies a Core Gene Set Related to Long-Term Memory. J Neurosci 37, 6661-6672, doi:10.1523/JNEUROSCI.3298-16.2017 (2017). 
33 Lakhina, V. et al. Genome-wide functional analysis of CREB/long-term memorydependent transcription reveals distinct basal and memory gene expression programs. Neuron 85, 330-345, doi:10.1016/j.neuron.2014.12.029 (2015).

34 Chew, Y. L., Grundy, L. J., Brown, A. E. X., Beets, I. \& Schafer, W. R. Neuropeptides encoded by nlp-49 modulate locomotion, arousal and egg-laying behaviours in Caenorhabditis elegans via the receptor SEB-3. Philosophical Transactions of the Royal Society B: Biological Sciences 373, doi:10.1098/rstb.2017.0368 (2018). 


\section{Figure legends:}

2 Figure 1: The neuropeptide FLP-20 is required for both habituation and sensitization. A)

3 Intermediate-term memory for habituation in response to mechanical stimulation (taps) after

4 massed training requires FLP-20 release from the touch receptor neurons (TRNs). ISI: Inter-

5 stimulus interval. B) Sensitization of escape responses mediated by the nociceptive neuron

6 ASH requires FLP-20 release from the touch neurons, which act through the FRPR-3 receptor

7 to activate the neuroendocrine cell RID. C) Schematic of specific neurons highlighted above:

8 six TRNs (ALML/R, AVM, PVM, PLML/R) in the body and tail, the RID interneuron and

9 ASH sensory neuron. For simplicity, only the soma of RID and ASH are shown.

11 Figure 2: Studies of neuropeptide signalling in associative learning in C. elegans indicate that

12 A) one neuropeptide can have modulatory effects on a variety of learned behaviours; B) a

13 specific learned behaviour can be modulated by multiple neuropeptides; C) learning circuits

14 are often modulated at different nodes by different neuropeptides; D) neuropeptides can

15 differentially modulate specific stages of the learning process. 